\title{
The Chronicles of Myelodysplastic/ Myeloproliferative Legacy - Chronic Myelomonocytic Leukemia
}

\author{
Vinu Sugathan ${ }^{1}$, Latha Abraham ${ }^{1}$ and Mobin Paul ${ }^{2 *}$ \\ ${ }^{1}$ Department of Pathology, Rajagiri Hospital \\ ${ }^{2}$ Department of Clinical Haematology \& Haemato-oncology, Rajagiri Hospital
}

\begin{abstract}
Chronic myelomonocytic leukemia is a heterogeneous syndrome with features of both myelodysplastic syndromes (MDS) and myeloproliferative neoplasms (MPN). The varied clinical presentations add to the distinctiveness of the disease. This heterogeneity should invigorate the search for reliable predictors of evolution and progression of the disease. Prior Indian research studies on CMML are limited. We report a case series from a tertiary care centre in Kerala, South India. This was a retrospective observational study of all cases of CMML, which was diagnosed in the departments of Pathology and Clinical Haematology \& Haemato- oncology of our institution between January 2017 to May 2020. The clinical presentation, laboratory investigations, and treatment details were noted from the electronic medical case records. Nine cases of CMML were encountered during the study period. The mean age of study subjects was 70.4 years with a female predilection. Fever and weight loss were the most common clinical presentations. Four patients were classified as CMML- 2, three patients as CMML- 1, and two as CMML- 0. Based on the WBC count, five patients were classified as dysplastic and four as proliferative subtypes. Two patients had grade $1 / 3$ (one case each of CMML- 2 and CMML- 1 ) and one patient had grade $2 / 3$ fibrosis (a case of CMML- 1 ) in the bone marrow. Thirty-three percentage patients had clonal cytogenetic abnormalities, the commonest being trisomy 8 . Renal function was deranged in three patients and two patients had a deranged liver function and hepatomegaly. Four patients underwent treatment with hypomethylating agents or cytoreduction with hydroxyurea. One of the patients (CMML- 2 with marked leucocytosis) succumbed to the disease.
\end{abstract}

Keywords: CMML, Monocytosis, Myelodysplastic, Myeloproliferative

\section{Introduction}

Chronic myelomonocytic leukemia (CMML) is a clonal hematopoietic stem cell neoplasm with overlapping features of myelodysplastic syndromes (MDS) and myeloproliferative neoplasms (MPN). CMML is characterized by abnormal proliferation of the cells of monocytic lineage and dysplasia in one or more hematopoietic cell lines. The disease poses an increased risk of transformation to secondary acute myeloid leukemia (AML). In the most recent update of the WHO 2016 classification, CMML is listed amongst the MDS/MPN overlap disorders. ${ }^{1} \mathrm{CMML}$ is now divided into CMML-0, CMML-1, and CMML-2 based on the percentage of blasts. Two CMML subtypes, myelodysplastic (MDS-CMML with total leukocyte count $\leq 13 \times 10^{9} / \mathrm{L}$ ) and myeloproliferative (MP-CMML with total leukocyte count $>13 \times 10^{9} / \mathrm{L}$ ), based on white blood cell count are also identified. The diagnosis of CMML now requires both an absolute monocytosis $\left(\geq 1 \times 10^{9} / \mathrm{L}\right)$ and relative monocytosis ( $\geq 10 \%$ of leukocytes) in the peripheral blood. As per definition, in the 2008 and 2016 updates of the WHO classification, CMML can only be diagnosed when rearrangements in PDGFRA, PDGFRB, or FGFR1 genes and the PCM1-JAK2 fusion gene have been excluded. ${ }^{2}$ The Philadelphia chromosome (BCR-
$A B L 1$ fusion gene product) is absent in CMML whereas, $J A K 2$ mutation $\mathrm{V} 617 \mathrm{~F}$ or the KIT mutation $\mathrm{D} 816 \mathrm{~V}$ may be detected. Mutations in SRSF2, TET2 or RAS are not diseasespecific, but are also detected in MDS, MPN or AML. ${ }^{3}$

Examination of the peripheral blood (PB) and bone marrow (BM) smears is crucial for diagnosis in a suspected CMML case. Myeloblasts, monoblasts, and promonocytes (blast equivalent) are included to estimate the blast percentage. Promonocytes have abundant light-grey to blue cytoplasm with scattered fine lilac granules and lacy nuclear chromatin with prominent nucleoli, and delicate nuclear convolutions. Abnormal monocytes have denser chromatin with more nuclear convolutions; and they are not included in the blast count. Dysgranulopoiesis including hyposegmented or abnormally segmented nuclei or abnormal cytoplasmic granulation is present in most cases. In some cases, it may be difficult to distinguish between hypogranular neutrophils and dysplastic monocytes. ${ }^{4}$

Cytochemical studies are useful in difficult cases. Alphanaphthyl butyrate esterase or alpha-naphthyl acetate esterase (with fluoride inhibition) staining in combination with naphthol AS-D chloroacetate esterase (CAE) staining, is useful for assessing the monocytic component. ${ }^{5}$ 
Flow cytometry studies are helpful to confirm the monocyte and blast cell counts in these patients and to exclude AML. The blood and marrow cells usually express typical myelomonocytic antigens (e.g. CD33 and CD13) and variably express CD14, CD68, and CD64. The monocytes often have two or more aberrant immunophenotypic features like decreased expression of CD14, HLADR, CD13, CD11c, CD15, CD16, CD64, and CD36; overexpression of CD56; aberrant expression of CD2. An increased proportion of $\mathrm{CD} 14+/ \mathrm{CD} 16-$ monocytes has recently been described. ${ }^{6}$ Maturing myeloid cells may also have aberrant immunophenotypic features. An increased proportion of CD34+ cells and an emerging blast population with an aberrant immunophenotype have been associated with early transformation to acute leukemia. For the identification of monocytic cells, immunohistochemistry on tissue sections is less sensitive than cytochemistry or flow cytometry.

Several specific scoring systems taking CMML-related features into account have also been proposed, which is preferred now, than the International Prognostic Scoring System (IPSS) which served as the gold standard for prognostication in MDS and dysplastic CMML until $2012 .{ }^{7}$ The existing prognostic models, incorporating molecular information include the Mayo Molecular Model (MMM) and the Groupe Francais des Myelodysplasies ( GFM ) model. The Mayo Molecular Model (MMM) classifies patients into four categories: low, intermediate- $1 / 2$, and high risk, with median OS (overall survival) of 97, 59, 37 and 16 months respectively. On the other hand, the GFM model risk stratifies patients into three categories; low, intermediate, and high risk, with a median OS of 56, 27.4 and 9.2 months respectively. The recently developed MayoFrench cytogenetic risk stratification system, classifies patients into three groups; high (complex and monosomal karyotype), intermediate (all karyotypes not belonging to high and low-risk groups) and low risk (normal, $-\mathrm{Y}$ and $\operatorname{del}(3 q)$ ), with a median OS of 3 months, 20 months and 41 months, respectively. ${ }^{8}$

The only curative option for CMML remains allogeneic stem cell transplantation and is therefore recommended for most young and eligible patients with acceptable transplantrelated risk. The management of myelodysplastic CMML is congenerous to myelodysplastic syndromes, resorting to the use of erythropoiesis-stimulating agents to cope with anemia in addition to careful monitoring and supportive care. On the other hand, the management of proliferative CMML usually relies on cytoreductive agents such as hydroxyurea. Hypomethylating agents are the preferred option in the presence of excessive blasts and other poor prognostic factors like cytopenias even though their impact on leukemic transformation and survival has not been proved. A subset of classical CMML patients may benefit from hypomethylating agents like 5-azacytidine and decitabine by providing long-term disease control. ${ }^{9}$

\section{Case Series}

A retrospective review of medical records of all patients who were evaluated and treated in the departments of clinical hematology \& hemato-oncology and pathology and fulfilled the criteria for CMML over a period of forty months from January 2017 to May 2020 was done. These patients were evaluated using complete blood count, peripheral smear, bone marrow aspiration, bone marrow biopsy, and cytogenetic/molecular studies.

There were a total number of nine patients $(n=9)$ ranging between 56 and 85 years of age with a mean age of 72.5 and a median of 78. Most of the patients were females $(\mathrm{n}=5)$. Four patients with total WBC count greater than 13,000/dl were classified as proliferative CMML and the rest five were dysplastic CMML. Those with proliferative CMML presented with fever, fatigue, leucocytosis, and hepatomegaly. Those with dysplastic CMML presented with fever, weight loss, bleeding symptoms, pallor, and hepatomegaly. All patients were anemic and four patients had thrombocytopenia. Total leucocyte counts were elevated in four patients and the remaining five had normal counts. All patients satisfied the criteria of absolute and relative monocytosis ranging from 1.1 to $13.9 \times 10^{6} / \mathrm{dl}$ (13 to $\left.42 \%\right)$. In our study six patients had grade $0 / 3$ fibrosis, two patients' grade $1 / 3$ (CMML- 2 and CMML-1) and one patient had grade 2/3 fibrosis (CMML- 1) in the bone marrow. Four patients had a normal karyotype, two had trisomy 8 , one patient had a deletion of $9 q$ and the $Y$ chromosome. BCR-ABL gene rearrangement was not detected in any of the patients. Five patients had LDH values above $200 \mathrm{IU} / \mathrm{L}$. Renal function was deranged in three patients and two patients with hepatomegaly had a deranged liver function. Four patients were classified as CMML- 2, three patients as CMML-1, and two as CMML- 0 .

Out of the nine patents, four patients received treatment. Two patients were treated with hydroxyurea (one case of CMML-2 and one case of CMML-0). Two patients (CMML -2) are being treated with azacytidine, and their blood cell counts improved over time. Two patients (one case of CMML-2 and one case of CMML -1) were lost to follow up. Other patients with CMML-1 and CMML-0 are on regular follow up. One of the patients (CMML-2 with marked leucocytosis) succumbed to the disease. 
Table 1: Clinical and laboratory parameters of study subjects

\begin{tabular}{|c|c|c|c|c|c|c|c|c|c|}
\hline Parameters & Case 1 & Case 2 & Case 3 & Case 4 & Case 5 & Case 6 & Case 7 & Case 8 & Case 9 \\
\hline Age (years) & 60 & 67 & 79 & 60 & 84 & 56 & 85 & 78 & 84 \\
\hline Sex & $F$ & $F$ & M & $F$ & M & $F$ & M & M & $F$ \\
\hline $\mathrm{HB}(\mathrm{gm} / \mathrm{dl})$ & 8.4 & 4 & 10.9 & 8.3 & 9.9 & 11.1 & 9.7 & 9 & 8.8 \\
\hline TC $\times 10^{9} / 1$ & 92.9 & 9.1 & 9.2 & 7 & 23.1 & 25.1 & 19.7 & 7.1 & 5.9 \\
\hline $\begin{array}{l}\text { Monocyte count } \times 10^{9} / 1 \\
\text { and } \%\end{array}$ & $\begin{array}{l}13.9 \\
15 \% \\
\end{array}$ & \begin{tabular}{|l|}
1.4 \\
$15 \%$ \\
\end{tabular} & \begin{tabular}{|l|}
1.3 \\
$13 \%$ \\
\end{tabular} & $\begin{array}{l}1.1 \\
20 \% \\
\end{array}$ & \begin{tabular}{|l|}
8.8 \\
$38 \%$ \\
\end{tabular} & \begin{tabular}{|l|}
4.8 \\
$19 \%$ \\
\end{tabular} & \begin{tabular}{|l|}
5.9 \\
$32 \%$ \\
\end{tabular} & $\begin{array}{l}1.2 \\
16 \% \\
\end{array}$ & $\begin{array}{l}2.5 \\
42 \% \\
\end{array}$ \\
\hline Platelet $\times 10^{9} / /$ & 158 & 120 & 120 & 270 & 207 & 435 & 80 & 40 & 171 \\
\hline PS blast (\%) & 3 & 2 & 0 & 1 & 0 & 2 & 0 & 0 & 8 \\
\hline BM blast (\%) & 16 & 12 & 8 & 5 & 1 & 13 & 1 & 2 & 16 \\
\hline $\mathrm{LDH}(\mathrm{IU} / \mathrm{L})$ & 371 & 245 & 201 & 132 & 212 & 248 & 194 & n/a & 266 \\
\hline RFT & $\mathrm{N}$ & $\mathrm{N}$ & $\mathrm{N}$ & Deranged & Deranged & $\mathrm{N}$ & Deranged & n/a & $\mathrm{N}$ \\
\hline LFT & $N$ & $\mathrm{~N}$ & $N$ & Deranged & $\mathrm{N}$ & $\mathrm{N}$ & $N$ & n/a & $\mathrm{N}$ \\
\hline BM Fibrosis (grade) & n/a & 0 & $1 / 3$ & $2 / 3$ & 0 & 0 & 0 & 0 & $1 / 3$ \\
\hline Subtype & CMML2 & CMML2 & CMML1 & CMML1 & CMML 0 & CMML2 & CMMLO & CMMLO & CMML2 \\
\hline MP-CMML/MD-CMML & MP & MD & MD & MD & MP & MP & MP & MD & MD \\
\hline Karyotype & $46 X X$ & $47 X X+8(10)$ & $46 X Y$ & $46 X X+8(20)$ & $46 X Y$ & $46 X X$ & $\begin{array}{l}\text { 45X-Y } \\
\text { DEL } 9 Q\end{array}$ & $46 X Y$ & $46 X X$ \\
\hline Treatment & Hydroxyurea & Lost follow up & Follow up & Lost follow up & Hydroxyurea & Azacytidine & Follow up & Follow up & Azacytidine \\
\hline
\end{tabular}

Abbreviations: $M$ - male, F-female, $N$-normal, n/a-not available, PS- peripheral smear, BM-bone marrow, LDH-lactate dehydrogenase, RFT-renal function test, LFT-liver function test, CMML- chronic myelomonocytic leukemia, MP- myeloproliferative, MD- myelodysplastic

Table 2: Comparison of various parameters of our study with other studies

\begin{tabular}{|c|c|c|c|}
\hline Parameters & Our study & Nabeel et al study ${ }^{10}$ & Mayo prognostic model study ${ }^{11}$ \\
\hline $\begin{array}{l}\text { Number of study } \\
\text { subjects }\end{array}$ & 9 & 9 & 226 \\
\hline Age in years (Range) & $56-85$ & $53-84$ & $20-90$ \\
\hline Sex & $4(\mathrm{M}), 5(\mathrm{~F})$ & $8(\mathrm{M}), 1(\mathrm{~F})$ & 152(M), $74(\mathrm{~F})$ \\
\hline Haemoglobin (gm\%) & $4-11.1$ & $5.3-12.4$ & $6.4-15.6$ \\
\hline Total WBC Count $\left(\mathrm{X} 10^{9} / \mathrm{I}\right)$ & $7-92.9$ & $3.7-88.3$ & $1.3-302$ \\
\hline $\begin{array}{l}\text { Absolute monocyte count } \\
\text { (X109/l)/percentage }\end{array}$ & $\begin{array}{l}1.1 \\
13-42 \% \\
\end{array}$ & $\begin{array}{l}\text { N/A } \\
11-44 \%\end{array}$ & $\begin{array}{l}1-40 \\
\text { N/A }\end{array}$ \\
\hline Platelet Count $\left(\mathrm{X} 10^{9} / \mathrm{L}\right)$ & $40-435$ & $20-206$ & $8-1100$ \\
\hline CMML subdivisions & $\begin{array}{l}\text { CMML-2(4) } \\
\text { CMML-1 (2) } \\
\text { CMML-0 (3) }\end{array}$ & $\begin{array}{l}\text { CMML-2(3) } \\
\text { CMML-1 (4) } \\
\text { CMML-0 (2) }\end{array}$ & $\begin{array}{l}\text { CMML-1 (191) } \\
\text { CMML2 (35) }\end{array}$ \\
\hline Karyotypic abnormalities (\%) & $33 \%$ & $0 \%$ & $30 \%$ \\
\hline LDH levels (IU/L) & $132-371$ & $482-831$ & $\mathrm{~N} / \mathrm{A}$ \\
\hline LFT derangements (\%) & $22 \%$ & $22 \%$ & $\mathrm{~N} / \mathrm{A}$ \\
\hline Deaths & 1 & 2 & 176 \\
\hline
\end{tabular}



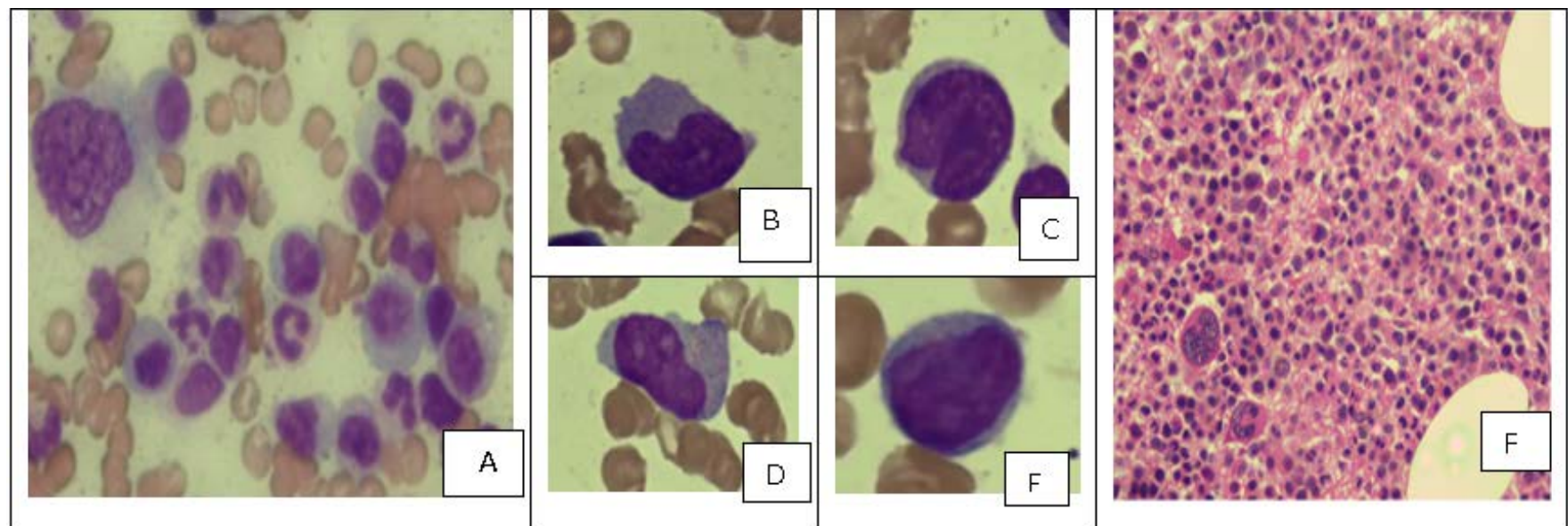

Fig. 1: Bone marrow aspirate and trephine biopsy.

A. Bone marrow aspiration (Leishman stain, 100X): Hypercellular marrow showing myeloid hyperplasia, monocytosis and dysgranulopoiesis

B. Monocyte (Leishman stain, 400X)

C. Promonocyte (Leishman stain, 400X)

D. Monoblast (Leishman stain, 400X)

E. Myeloblast (Leishman stain, 400X)

F. Bone marrow trephine biopsy (Hematoxylin\& Eosin stain, 400X): Solidly cellular marrow with myeloid hyperplasia
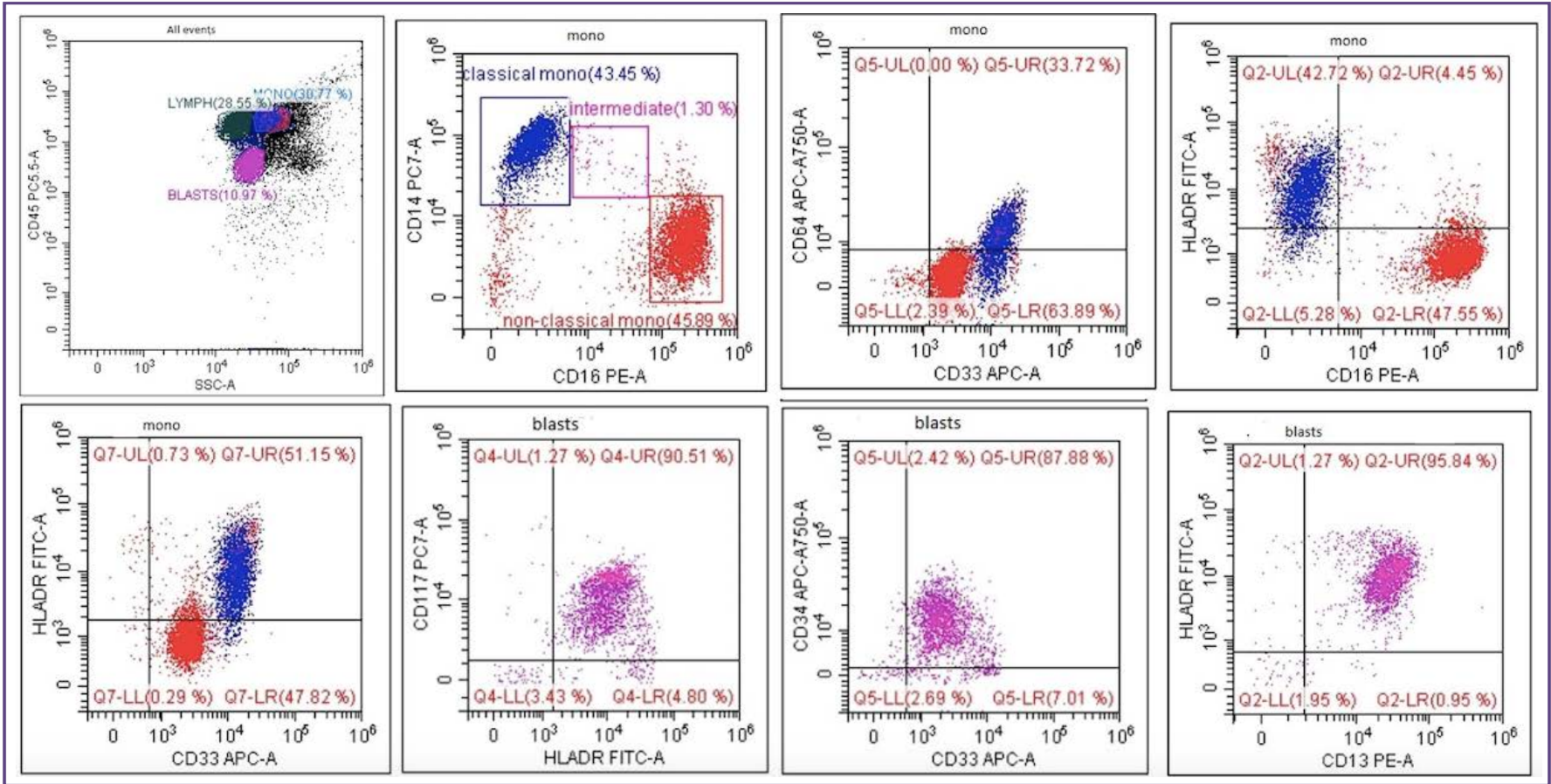

Fig. 2: Flow cytometric dot plot in a case of CMML- 2, Flow cytometric dot plot showing around 10\% CD45 dim population (blasts -pink) expressing CD13, CD33, HLADR, CD34 and CD117. Around 35\% CD45 bright monocyte population is also present with 43\% classical monocytes (blue - CD14+CD16-) and 46\% non-classical monocytes (red- CD14dim/-CD16++). Non classical monocytes are negative for CD64 and HLADR and dim CD33. 


\section{Discussion}

Theagerange ofourseries was from 56 to 85 years with a mean age of 72.5 and a median of 78. A female predilection was seen. Another Indian case series by Nabeel et al has recorded a median age of 67.4 years with a male predilection..$^{10}$ The two large studies- the Mayo Clinic study and the Groupe Francais des Myelodysplasies (GFM) study report an age range of 20 to 90 years and 40 to 91 years respectively. The median age of presentation of various studies ranges between $71-74$ years, with a male predilection. ${ }^{11}$ Most of the patients presented with fever and weight loss in our study. Those with proliferative CMML presented with fever, fatigue, leucocytosis, and hepatomegaly. Those with dysplastic CMML presented with fever, weight loss, bleeding symptoms, pallor, and hepatomegaly. The clinical presentation of patients with CMML is varied and the heterogeneity underlies the basis for the current categorization into MDS- CMML and MPN-CMML. ${ }^{12}$ Those with a dysplastic phenotype tend to present with cytopenias, easy bruising and fatigue, recurrent infections, and transfusion dependence. Proliferative phenotype is characterized by an increased total WBC count, monocytosis, and organomegaly. The common clinical presentations include fatigue, night sweats, bone pains, weight loss, and cachexia. ${ }^{3}$

Increased blast count, low haemoglobin, raised serum lactate dehydrogenase (LDH), and possibly an increased lymphocyte count is the most important independent prognostic parameters as seen in multivariate analyses performed by several groups. Karyotype analysis was not customarilyshowntoyieldadditional prognosticinformation. Renal function was deranged in three patients and two patients had a deranged liver function and hepatomegaly. Renal impairment from CMML is uncommon. Patients present with renal failure haematuria, renal or perirenal haemorrhage secondary to associated vasculitis, or renal infarction secondary to blast crisis. Hepatomegaly is uncommon particularly with isolated elevated ALP and is associated with poor prognosis. ${ }^{13}$ Our study showed $33 \%$ of patients having clonal cytogenetic abnormalities, the commonest being trisomy 8 . According to current literature $30 \%$ of CMML patients have clonal cytogenetic abnormalities. The most common cytogenetic abnormalities include trisomy $8(+8)$, deletion of the $\mathrm{Y}$ chromosome (-Y), chromosome 7 abnormalities (monosomy 7 and del 7q), trisomy $21(+21)$, and complex karyotypes. ${ }^{14}$ In our study six patients had grade $0 / 3$ fibrosis, two patients had grade 1/3 (CMML- 2 and CMML-1) and one patient had grade 2/3 fibrosis (CMML-1) in the bone marrow. None of the cases had collagen fibrosis. Patients with any amount of BM fibrosis (MF-1 or higher; MF1+) were found to have significantly shorter progression-free survival, splenomegaly, and increased BM megakaryocytes compared to patients without BM fibrosis (MF-0). Peripheral blood parameters, presence of JAK2 V617F mutation, BM blasts, and overall survival did not show any association with fibrosis. ${ }^{15}$

\section{Conclusions}

CMML is a rare hematopoietic stem cell neoplasm with overlapping features of MDS and MPN. A median age of 78 years with a slight female predilection was observed. Most of the patients presented with fever, weight loss, and cytopenias. Four cases of CMML 2, three cases of CMML 1, and two cases of CMML0 were identified. Thirty-three percent of patients had clonal cytogenetic abnormalities commonest being trisomy 8 and deletion Y. Renal function was deranged in three patients and two patients had a deranged liver function and hepatomegaly. The limited number of cases diagnosed within the relatively long study period of forty months is a reflection of the fact that this is an uncommon disease in the general population. Clinical features, prognostic parameters, and survival vary among individuals; which justifies a need for personalized treatment approach, to improve the quality of life, particularly in the elderly. This study adds to the clinicopathologic data profile of the disease.

\section{Abbreviations}

CMML - Chronic Myelomonocytic Leukemia

MDS - Myeodysplastic Syndrome

MPN - Myeloproliferative Neoplasm

AML - Acute Myeloid Leukemia

PB - Peripheral Blood

BM - Bone Marrow

RFT -Renal Function Test

LFT- Liver Function Test

HXU -Hydroxyurea

AZD- Azacytidine

N- Normal

\section{Acknowledgments}

We thank Dr. Ansu Abu Alex for the flowcytometry plots.

\section{References}

1. Arber DA, Orazi A, Hasserjian R, et al. The 2016 revision to the World Health Organization classification of myeloid neoplasms and acute leukemia. Blood. 2016;127(20):23912405 . 
2. Patnaik MM, Parikh SA, Hanson CA, Tefferi A. Chronic myelomonocytic leukaemia: a concise clinical and pathophysiological review. Br J Haematol. 2014;165(3):273286.

3. Kohlmann A, Grossmann V, Klein HU, et al. Next-generation sequencing technology reveals a characteristic pattern of molecular mutations in $72.8 \%$ of chronic myelomonocytic leukemia by detecting frequent alterations in TET2, CBL, RAS, and RUNX1. J Clin Oncol. 2010;28(24):3858-3865.

4. Martiat P, Michaux JL, Rodhain J (1991). Philadelphia -negative (Ph-) chronic myeloidleukemia (CML): comparison with $\mathrm{Ph}+\mathrm{CML}$ and chronic myelomonocytic leukemia. The Groupe Francais de Cytogenetique Hematologique. Blood. 78:205- 11.

5. Orazi A, Chiu R, O'Malley DP, et al.(2006). Chronic myelomonocytic leukemia: The role of bone marrow biopsy immunohistology.Mod Pathol. 19:1536-45.

6. Selimoglu-Buet D, Wagner-Ballon 0, Saada V, et al. (2015). Characteristic repartition of monocyte subsets as a diagnostic signature of chronic myelomonocytic leukemia. Blood. 125:3618-26.

7. Greenberg $\mathrm{P}, \mathrm{Cox} \mathrm{C}$, LeBeau MM, et al International scoring system for evaluating prognosis in myelodysplastic syndromes. Blood. 1997;89(6):2079-2088

8. Padron E, Garcia-Manero G, Patnaik MM, et al. An international data set for CMML validates prognostic scoring systems and demonstrates a need for novel prognostication strategies. Blood Cancer J. 2015; 5:e333

9. Hunter AM, Zhang L, Padron E. Current Management and Recent Advances in the Treatment of Chronic
Myelomonocytic Leukemia. Curr Treat Options Oncol. 2018;19(12):67. Published 2018 Oct 27. doi:10.1007/ s11864-018-0581-6

10. Nabeel Azeez K, Venkatesan Somasundaram, Isha Sharma , Sanjeevan Sharma and Ajay Malik .Clinicopathological Profile of Chronic Myelomonocytic Leukemia Cases: An Experience from A Tertiary Care Center, Annals of Pathology and Laboratory Medicine, Vol. 6, Issue 10, October, 2019, eISSN: 2349-6983; pISSN: 2394-6466

11. Patnaik MM, Wassie EA, Padron E, et al. Chronic myelomonocytic leukemia in younger patients: molecular and cytogenetic predictors of survival and treatment outcome [published correction appears in Blood Cancer J. 2015;5:e280] [published correction appears in Blood Cancer J. 2015;5:e280]. Blood Cancer J. 2015;5(1):e270.

12. Bennett JM, Catovsky D, Daniel MT, et al. Proposals for the classification of the acute leukaemias. FrenchAmerican-British (FAB) co-operative group. $\mathrm{Br}$ JHaematol. 1976;33:451-458

13. Germing U, Kündgen A, GattermannN. Risk assessment in chronic myelomonocytic leukemia(CMML). Leuk Lymphoma. 2004;45(7):1311-1318.

14. Patnaik MM, Tefferi A. Cytogenetic and molecular abnormalities in chronic myelomonocytic leukemia. Blood Cancer J. 2016; 6:e 393.

15. Petrova-Drus, K., Chiu, A., Margolskee, E., BaroukFox, S., Geyer, J., Dogan, A., \& Orazi, A. (2017). Bone marrow fibrosis in chronic myelomonocytic leukemia is associated with increased megakaryopoiesis, splenomegaly and with a shorter median time to disease progression. Oncotarget, 8(61), 103274-103282.

*Corresponding author:

Mobin Paul, MBBS, MD,DM., Consultant - Clinical Haematologist \& Haemato-oncologist, Department of Clinical Haematology \& Haemato-oncology, In charge - Blood \& Marrow Stem cell transplant services., Rajagiri Hospital.Near GTN Junction, Chunangamvely, Alwaye, Kochi, Kerala 683112 Phone: +918903232476

Email: mobinpau199@gmail.com

Date of Submission : 09/08/2019

Financial or other Competing Interests: None. 